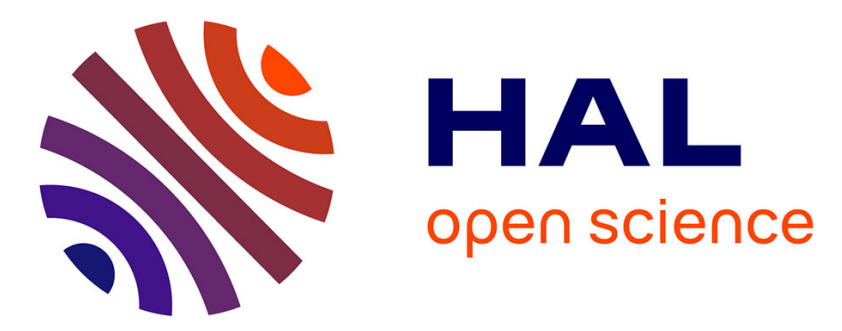

\title{
Preformation of clusters in heavy nuclei and cluster radioactivity
}

H.F. Zhang, J.M. Dong, Guy Royer, W. Zuo, J.Q. Li

\section{To cite this version:}

H.F. Zhang, J.M. Dong, Guy Royer, W. Zuo, J.Q. Li. Preformation of clusters in heavy nuclei and cluster radioactivity. Physical Review C, 2009, 80, pp.037307. 10.1103/PhysRevC.80.037307 . in2p3-00420548

\section{HAL Id: in2p3-00420548 https://hal.in2p3.fr/in2p3-00420548}

Submitted on 29 Sep 2009

HAL is a multi-disciplinary open access archive for the deposit and dissemination of scientific research documents, whether they are published or not. The documents may come from teaching and research institutions in France or abroad, or from public or private research centers.
L'archive ouverte pluridisciplinaire $\mathbf{H A L}$, est destinée au dépôt et à la diffusion de documents scientifiques de niveau recherche, publiés ou non, émanant des établissements d'enseignement et de recherche français ou étrangers, des laboratoires publics ou privés. 


\title{
Preformation of clusters in heavy nuclei and cluster radioactivity
}

\author{
H.F. Zhang ${ }^{1,4},{ }^{*}$ J.M. Dong ${ }^{1}$, G. Royer ${ }^{2}$, W. Zuo ${ }^{1,3,4}$, and J.Q. Li ${ }^{1,3,4}$ \\ ${ }^{1}$ School of Nuclear Science and Technology, Lanzhou University, Lanzhou 730000, People's Republic of China \\ 2 Laboratoire Subatech, UMR: IN2P3/CNRS-Université-Ecole des Mines, Nantes 44, France \\ ${ }^{3}$ Institute of Modern Physics, Chinese Academy of Science, Lanzhou 730000, People's Republic of China and \\ ${ }^{4}$ Kavli institute for Theoretical Physics China, Chinese Academy of Sciences, Beijing 100190, People's Republic of China
}

Within the preformed cluster model approach, the values of the preformation factors have been deduced from the experimental cluster decay half-lives assuming that the decay constant of the heavy-ion emission is the product of the assault frequency, the preformation factor and the penetrability. The law according to which the preformation factors follow a simple dependence on the mass of the cluster was confirmed. Then predictions for some most possible cluster decays are provided.

PACS numbers: 23.70. $+\mathrm{j}, 21.10 . \mathrm{Jx}, 27.90 .+\mathrm{b}$

Cluster radioactivity (heavy-ion radioactivity) by heavy nuclei with an emitted cluster heavier than $\alpha$ particle but lighter than fission fragments was firstly theoretically predicted in the beginning of 1980's by Sandulescu, Poenaru and Greiner [1]. The first observation was the detection of ${ }^{14} \mathrm{C}$ emitted from ${ }^{223} \mathrm{Ra}$ by Rose and Jones [2]. Since then, other cluster radioactivities have been observed leading to ${ }^{14} \mathrm{C},{ }^{20} \mathrm{O},{ }^{23} \mathrm{~F}{ }^{22,24-26} \mathrm{Ne}$, ${ }^{28,30} \mathrm{Mg}$ and ${ }^{32,34} \mathrm{Si}$ emission and their partial half-lives have been measured. The decaying parent nuclei range from ${ }^{221} \mathrm{Fr}$ to ${ }^{242} \mathrm{Cm}$ at present, all from the trans-lead region, while the daughter nuclei are almost closed shell spherical nuclei. This indicates that shell effects play a key role to select possible cluster emissions and the study of cluster emission can be used to identify shell effects including the very weak sub-shell closures [3-5]. Several theoretical approaches can be employed to investigate cluster emission: among them the preformed cluster model (PCM) [3, 5, 6], in which the cluster is assumed to be preformed in the parent nucleus and the preformation factor for all possible clusters is calculated by solving the Schrödinger equation for the dynamical flow of mass and charge; the super-asymmetric fission model [7-11], which is based on the Gamow's idea of barrier penetration; the unified fission model [12-14](some authors name it the Coulomb and proximity potential model); a cluster model with a mean-field cluster potential can also provide a good description of cluster emission [15].

In the present work, the cluster decay constant is the product of the assault frequency, the preformation factor and the penetrability. The potential barrier which governs the heavy-ion emission has been determined using the generalized liquid drop model with the help of the experimental $\mathrm{Q}$ value. The GLDM describes the shape evolution from one body to two separated fragments in an unified way. The preformation factor has been extracted from the experimental cluster decay half-lives and from the theoretical determination of the penetrability and the usual assault frequency. As long as the relation between

\footnotetext{
*Electronic address: zhanghongfei@lzu.edu.cn
}

the preformation factor and the cluster decay is valuable, predictions can be given for the possible cluster decays using the $\mathrm{Q}$ value from Audi's recent data [16].

The cluster decay constant is defined as [17]:

$$
\lambda=P_{0} \nu_{0} P .
$$

Imagining the cluster moving back and forth inside the nucleus with a velocity $v=\sqrt{2 E / M}$, it presents itself at the barrier with a frequency $\nu_{0}=\frac{1}{2 R} \sqrt{2 E / M} . R$ is the radius of the parent nucleus and $E$ is the energy of the cluster, corrected for recoil; $M$ is the average value of mass inertia for the cluster, which will be discussed later. The penetration probability $P$ is calculated within the WKB approximation. The potential barrier governing the cluster emission is determined within the GLDM $[10$, 17 .

The barrier penetrability $P$ is calculated within the action integral

$$
P=\exp \left[-\frac{2}{\hbar} \int_{R_{\mathrm{in}}}^{R_{\mathrm{out}}} \sqrt{2 B(r)(E(r)-E(\text { sphere }))} d r\right],
$$

where $R_{\text {in }}$ is the distance between the mass centres of the portions of the initial sphere separated by a plane perpendicular to the deformation axis to assume volume conservation of the future fragments. $R_{\text {out }}$ is simply $e^{2} \mathrm{Z}_{d} \mathrm{Z}_{c} / \mathrm{Q}$. The inertia has been chosen as $B(r)=\mu(1+1.3 f(r))[11]$ where

$$
f(r)= \begin{cases}\sqrt{\frac{R_{\text {cont }}-r}{R_{\text {cont }}-R_{i n}}}, & r \leq R_{\text {cont }} \\ 0, & r \geq R_{\text {cont }}\end{cases}
$$

$R_{\text {cont }}=R_{1}+R_{2}, R_{1}$ and $R_{2}$ are the radii of daughter nucleus and cluster respectively. The present inertia can simulate a rapid variation of the friction force effects only at the moment of the neck rupture between the nascent fragments. The preformation factor $P_{0}$ of a cluster inside the mother nucleus can be estimated from Eq.(1).

The resulting potential barriers for $\alpha$ decay and cluster ${ }^{32} \mathrm{Si}$ emission of ${ }^{238} \mathrm{Pu}$ are displayed in Fig. 1 and Fig. 2, respectively. The maximum of the pure Coulomb barrier lies at the touching point between the nascent fragments. 


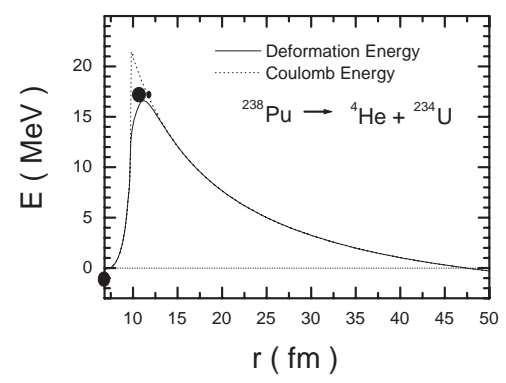

FIG. 1: Potential barrier of $\alpha$ emission from ${ }^{238} \mathrm{Pu}$ with (solid line) and without (dotted line) the proximity energy.

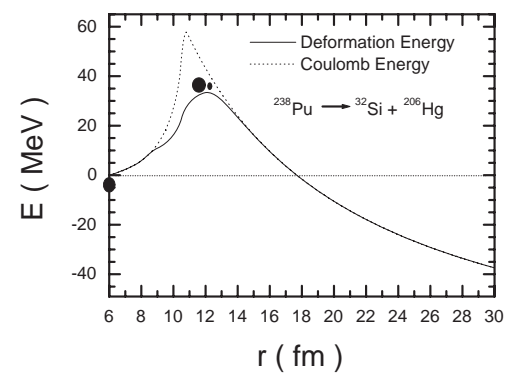

FIG. 2: Potential barrier of ${ }^{32} \mathrm{Si}$ emission from ${ }^{238} \mathrm{Pu}$ with (solid line) and without (dotted line) the proximity energy.

The introduction of the proximity forces lower the barrier of $4.9 \mathrm{MeV}$ for ${ }^{238} \mathrm{Pu} \rightarrow \alpha+{ }^{234} \mathrm{U}$, and $24.4 \mathrm{MeV}$ for ${ }^{238} \mathrm{Pu}$ $\rightarrow{ }^{32} \mathrm{Si}+{ }^{206} \mathrm{Hg}$. Further, the peak is shifted towards a more external position. For cluster emission, the barrier is lowered by the proximity forces more than that for $\alpha$ decay since the asymmetry is weaker.

In order to estimate the contributions of the proximity forces on the cluster emission as well as on the $\alpha$ decay quantitatively, we calculated the penetrability with and without the proximity energy respectively. The results are presented in the third column of Tab. I. The ratio of the penetrabilities with and without the proximity energy $\left(P^{\text {Def }} / P^{\text {Coul }}\right)$ stands between 2 and 3 for the $\alpha$ decay and increases rapidly with the mass number of the emitted cluster.

The preformation factor may be considered as the overlap of the actual ground state configuration and the configuration representing the cluster coupled to the ground state of the daughter. Obviously it is expected to be much less than unity. The extracted preformation factors from the GLDM are listed in columns 5 of Tab. I, and the results from the DDM3Y model [18] are also listed in the last column for comparison. As can be seen, the preformation factors decrease with the increase of the emitted cluster mass number $A_{2}$. Our results are comparable to other values. For example, our calculated value
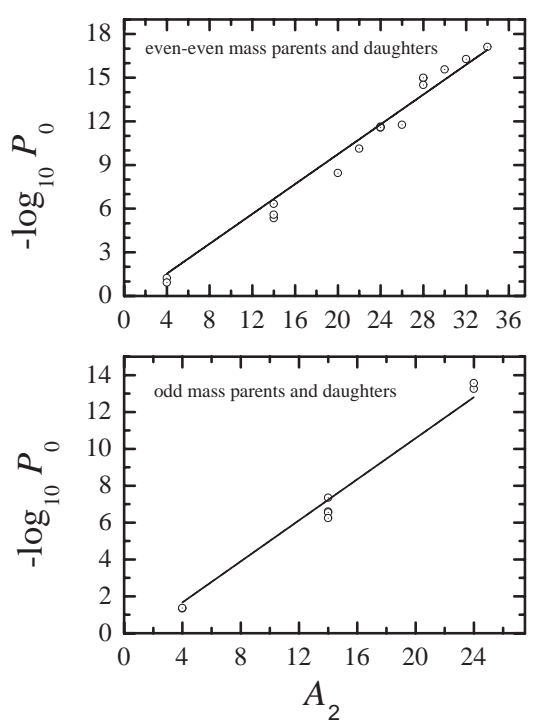

FIG. 3: Negative of logarithm of preformation factors $\left(\mathrm{P}_{0}\right)$ as a function of the cluster mass number $\mathrm{A}_{2}$.

of $P_{0}$ for ${ }^{212} \mathrm{Po}$ alpha emission is $3.405 \times 10^{-2}$ to be compared with $1.88 \times 10^{-2}[18]$ and $2.5 \times 10^{-2}$ deduced in [19]. A value of $3.1 \times 10^{-2}$ was obtained by Mohr [20] in a double folding model calculation using density from experimentally known charge distribution. The general coincidence of the preformation factors from the present results and the DDM3Y calculations is clear.

It has been suggested [21] that, in the case of heavy cluster decay, the preformation factor may scale as

$$
P_{0}=\left(P_{0}^{\alpha}\right)^{\left(A_{2}-1\right) / 3},
$$

where $A_{2}$ is the mass of the cluster and $P_{0}^{\alpha}$ is the preformation factor for the $\alpha$-decay. Thus a plot of $\log _{10} P_{0}$ against $\mathrm{A}_{2}$ should be a straight line. In the upper panel of Fig. 3, the negatives of $\log _{10} P_{0}$ as well as a best fit line are plotted for decays where both the parent and the daughter are even-even nuclei against the mass number of the cluster. The points fall nearly on a straight line with the $P_{0}^{\alpha}$ value given by $2.897 \times 10^{-2}$. This is comparable to the values $1.93 \times 10^{-2}$ by Bhattacharya and Gangopadhyay [18] and $1.61 \times 10^{-2}$ by Poenaru et al [22]. The study has been extended to decays where both the parent and the daughter nuclei have odd mass though the number of observed decays is rather small. The corresponding curve is shown in the lower panel of Fig. 3. Here the $S_{\alpha}$ value is $P_{0}^{\alpha}=0.0214$ which is very close to the value $P_{0}^{\alpha}=0.0135$ obtained in [18]. With such a linear fit of the logarithm of the spectroscopic factors with mass numbers, we have extended our scheme to calculate the half lives of some other possible decays where unambiguous lifetime measurements are not yet available and where possibilities of some other decays exist. The 
TABLE I: Preformation factor $P_{0}$ of cluster decay obtained in the present calculation and compared with that of the DDM3Y. The decay energy $Q$ and half-life $T$ are measured in $\mathrm{MeV}$ and s, respectively.

\begin{tabular}{|c|c|c|c|c|c|}
\hline$\overline{\overline{\text { Decay }}}$ & $\overline{\overline{Q_{\text {expt }}}}$ & $\overline{\mathrm{P}^{\text {Def }} / \mathrm{P}^{\text {Coul }}}$ & $\overline{\log _{10} T_{\text {expt }}}$ & $\overline{P_{0}^{\text {GLDM }}}$ & $P_{0}^{\text {DDM3Y }}$ \\
\hline${ }^{212} \mathrm{Po} \rightarrow{ }^{4} \mathrm{He}+{ }^{208} \mathrm{~Pb}$ & 8.950 & 2.80 & -6.52 & $3.405 \times 10^{-2}$ & $1.88 \times 10^{-2}$ \\
\hline${ }^{213} \mathrm{Po} \rightarrow{ }^{4} \mathrm{He}+{ }^{209} \mathrm{~Pb}$ & 8.540 & 2.78 & -5.37 & $2.652 \times 10^{-2}$ & $1.67 \times 10^{-2}$ \\
\hline${ }^{214} \mathrm{Po} \rightarrow{ }^{4} \mathrm{He}+{ }^{210} \mathrm{~Pb}$ & 7.833 & 2.45 & -3.78 & $8.734 \times 10^{-2}$ & $3.45 \times 10^{-2}$ \\
\hline${ }^{215} \mathrm{At} \rightarrow{ }^{4} \mathrm{He}+{ }^{211} \mathrm{Bi}$ & 8.178 & 2.50 & -4.00 & $2.467 \times 10^{-2}$ & $1.31 \times 10^{-2}$ \\
\hline${ }^{238} \mathrm{Pu} \rightarrow{ }^{4} \mathrm{He}+{ }^{206} \mathrm{U}$ & 5.59 & 2.14 & 9.59 & $1.462 \times 10^{-1}$ & \\
\hline${ }^{221} \mathrm{Fr} \rightarrow{ }^{14} \mathrm{C}+{ }^{207} \mathrm{Tl}$ & 31.317 & 54.71 & 14.52 & $2.559 \times 10^{-7}$ & $1.50 \times 10^{-8}$ \\
\hline${ }^{221} \mathrm{Ra} \rightarrow{ }^{14} \mathrm{C}+{ }^{207} \mathrm{~Pb}$ & 32.396 & 57.66 & 13.39 & $2.808 \times 10^{-7}$ & $1.55 \times 10^{-8}$ \\
\hline${ }^{222} \mathrm{Ra} \rightarrow{ }^{14} \mathrm{C}+{ }^{208} \mathrm{~Pb}$ & 33.05 & 64.23 & 11.00 & $4.619 \times 10^{-6}$ & $1.64 \times 10^{-7}$ \\
\hline${ }^{223} \mathrm{Ra} \rightarrow{ }^{14} \mathrm{C}+{ }^{209} \mathrm{~Pb}$ & 31.829 & 57.50 & 15.20 & $4.545 \times 10^{-8}$ & $2.85 \times 10^{-9}$ \\
\hline${ }^{224} \mathrm{Ra} \rightarrow{ }^{14} \mathrm{C}+{ }^{210} \mathrm{~Pb}$ & 30.54 & 58.36 & 15.92 & $2.614 \times 10^{-6}$ & $1.04 \times 10^{-7}$ \\
\hline${ }^{225} \mathrm{Ac} \rightarrow{ }^{14} \mathrm{C}+{ }^{211} \mathrm{Bi}$ & 30.477 & 35.58 & 17.34 & $5.743 \times 10^{-7}$ & $8.14 \times 10^{-8}$ \\
\hline${ }^{226} \mathrm{Ra} \rightarrow{ }^{14} \mathrm{C}+{ }^{212} \mathrm{~Pb}$ & 28.20 & 38.21 & 21.34 & $4.789 \times 10^{-7}$ & $3.97 \times 10^{-8}$ \\
\hline${ }^{228} \mathrm{Th} \rightarrow{ }^{20} \mathrm{O}+{ }^{208} \mathrm{~Pb}$ & 44.72 & 108.54 & 20.72 & $3.546 \times 10^{-9}$ & $8.37 \times 10^{-11}$ \\
\hline${ }^{230} \mathrm{U} \rightarrow{ }^{22} \mathrm{Ne}+{ }^{208} \mathrm{~Pb}$ & 61.40 & 166.04 & 19.57 & $7.537 \times 10^{-11}$ & $6.72 \times 10^{-12}$ \\
\hline${ }^{230} \mathrm{Th} \rightarrow{ }^{24} \mathrm{Ne}+{ }^{206} \mathrm{Hg}$ & 57.571 & 168.30 & 24.64 & $2.310 \times 10^{-12}$ & $1.87 \times 10^{-13}$ \\
\hline${ }^{231} \mathrm{~Pa} \rightarrow{ }^{24} \mathrm{Ne}+{ }^{207} \mathrm{Bi}$ & 60.417 & 210.29 & 23.38 & $5.542 \times 10^{-14}$ & $3.13 \times 10^{-15}$ \\
\hline${ }^{232} \mathrm{U} \rightarrow{ }^{24} \mathrm{Ne}+{ }^{208} \mathrm{~Pb}$ & 62.31 & 245.73 & 20.40 & $2.173 \times 10^{-12}$ & $9.77 \times 10^{-14}$ \\
\hline${ }^{233} \mathrm{U} \rightarrow{ }^{24} \mathrm{Ne}+{ }^{209} \mathrm{~Pb}$ & 60.486 & 228.12 & 24.82 & $2.725 \times 10^{-14}$ & $1.47 \times 10^{-15}$ \\
\hline${ }^{234} \mathrm{U} \rightarrow{ }^{24} \mathrm{Ne}+{ }^{210} \mathrm{~Pb}$ & 58.826 & 215.39 & 25.25 & $2.630 \times 10^{-12}$ & $1.54 \times 10^{-13}$ \\
\hline${ }^{233} \mathrm{U} \rightarrow{ }^{25} \mathrm{Ne}+{ }^{208} \mathrm{~Pb}$ & 60.776 & 330.45 & 24.82 & $3.276 \times 10^{-14}$ & $4.02 \times 10^{-16}$ \\
\hline${ }^{234} \mathrm{U} \rightarrow{ }^{26} \mathrm{Ne}+{ }^{208} \mathrm{~Pb}$ & 59.466 & 239.18 & 25.07 & $1.755 \times 10^{-12}$ & $1.67 \times 10^{-14}$ \\
\hline${ }^{234} \mathrm{U} \rightarrow{ }^{28} \mathrm{Mg}+{ }^{206} \mathrm{Hg}$ & 74.11 & 296.11 & 25.74 & $1.106 \times 10^{-15}$ & $6.30 \times 10^{-17}$ \\
\hline${ }^{236} \mathrm{Pu} \rightarrow{ }^{28} \mathrm{Mg}+{ }^{208} \mathrm{~Pb}$ & 79.67 & 464.31 & 21.67 & $1.029 \times 10^{-15}$ & $2.83 \times 10^{-17}$ \\
\hline${ }^{238} \mathrm{Pu} \rightarrow{ }^{28} \mathrm{Mg}+{ }^{210} \mathrm{~Pb}$ & 75.912 & 395.01 & 25.70 & $3.206 \times 10^{-15}$ & $1.21 \times 10^{-16}$ \\
\hline${ }^{238} \mathrm{Pu} \rightarrow{ }^{32} \mathrm{Si}+{ }^{206} \mathrm{Hg}$ & 91.19 & 944.18 & 25.28 & $5.343 \times 10^{-17}$ & $2.34 \times 10^{-18}$ \\
\hline${ }^{238} \mathrm{Pu} \rightarrow{ }^{30} \mathrm{Mg}+{ }^{208} \mathrm{~Pb}$ & 77.00 & 435.97 & 25.67 & $2.733 \times 10^{-16}$ & $2.34 \times 10^{-18}$ \\
\hline${ }^{242} \mathrm{Cm} \rightarrow{ }^{34} \mathrm{Si}+{ }^{208} \mathrm{~Pb}$ & 96.509 & 851.74 & 23.15 & $7.754 \times 10^{-18}$ & $1.10 \times 10^{-19}$ \\
\hline
\end{tabular}

results obtained with the GLDM and the fitted values of $P_{0}$ from Eq. (4) are tabulated in Tab. II, compared with the results of DDM3Y [18] and the upper limits on experimental half lives. Except in the case of the ${ }^{233} \mathrm{U}$ decay, the results from the two models are all consistent with the experimental observations. In the case of ${ }^{233} \mathrm{U}$, the results from GLDM and DDM3Y are very coincident, and the discrepancy between theory and experiment is small.

New possible islands of cluster emitters around the doubly magic nucleus ${ }^{100} \mathrm{Sn}$ and in the proton and neutron ranges $\mathrm{Z}=56-64$ and $\mathrm{N}=58-72$ respectively have been predicted [23-25]. The first experiment has concluded to the nonobservation of ${ }^{12} \mathrm{C}$ emission by ${ }^{114} \mathrm{Ba}$ [26]. The predictions for cluster decay half lives are presented in Tab. III, which may be useful for future experiments.

To summarize, the heavy ion emission from heavy nuclei has been studied within a preformed cluster approach and the GLDM. The decay constants are obtained from the experimental half-lives. The penetration probabilities are calculated from the WKB approximation and through the potential barriers determined with the GLDM. After using a classical method to estimate the assault frequencies the preformation factors are extracted systematically. Clearly the closed shell structures
TABLE II: Half-lives of cluster decay obtained with the GLDM and compared with the results of the DDM3Y and experimental data.

\begin{tabular}{|c|c|c|c|c|}
\hline$\overline{\text { Decay }}$ & $\begin{array}{c}Q \\
(\mathrm{MeV})\end{array}$ & $\begin{array}{c}\log _{10} T(\mathrm{~s} \\
\text { expt. }\end{array}$ & $\begin{array}{c}\log _{10} T(\mathrm{~s} \\
\text { GLDM }\end{array}$ & $\begin{array}{c}\log _{10} T(\mathrm{~s}) \\
\text { DDM3Y }\end{array}$ \\
\hline${ }^{223} \mathrm{Ac} \rightarrow{ }^{14} \mathrm{C}+{ }^{209} \mathrm{Bi}$ & 33.065 & & 13.738 & \\
\hline${ }^{223} \mathrm{Ac} \rightarrow{ }^{15} \mathrm{~N}+{ }^{208} \mathrm{~Pb}$ & 39.474 & & 14.806 & \\
\hline${ }^{224} \mathrm{Th} \rightarrow{ }^{14} \mathrm{C}+{ }^{210} \mathrm{Po}$ & 32.929 & & 14.289 & 13.68 \\
\hline${ }^{226} \mathrm{Th} \rightarrow{ }^{14} \mathrm{C}+{ }^{212} \mathrm{Po}$ & 30.596 & & 18.461 & 18.28 \\
\hline${ }^{224} \mathrm{Th} \rightarrow{ }^{16} \mathrm{O}+{ }^{208} \mathrm{~Pb}$ & 46.481 & & 15.590 & 15.47 \\
\hline${ }^{226} \mathrm{Th} \rightarrow{ }^{18} \mathrm{O}+{ }^{208} \mathrm{~Pb}$ & 45.727 & $>16.8$ & 18.381 & 18.23 \\
\hline${ }^{232} \mathrm{Th} \rightarrow{ }^{24} \mathrm{Ne}+{ }^{208} \mathrm{Hg}$ & 54.497 & $>29.2$ & 29.654 & 29.96 \\
\hline${ }^{236} \mathrm{U} \rightarrow{ }^{24} \mathrm{Ne}+{ }^{212} \mathrm{~Pb}$ & 55.945 & $>25.9$ & 29.971 & 30.16 \\
\hline${ }^{232} \mathrm{Th} \rightarrow{ }^{26} \mathrm{Ne}+{ }^{206} \mathrm{Hg}$ & 55.964 & $>29.2$ & 28.971 & 28.57 \\
\hline${ }^{233} \mathrm{U} \rightarrow{ }^{28} \mathrm{Mg}+{ }^{205} \mathrm{Hg}$ & 74.226 & $>27.6$ & 25.678 & 26.56 \\
\hline${ }^{237} \mathrm{~Np} \rightarrow{ }^{30} \mathrm{Mg}+{ }^{207} \mathrm{Bi}$ & 74.817 & $>27.6$ & 27.671 & 27.92 \\
\hline${ }^{240} \mathrm{Pu} \rightarrow{ }^{34} \mathrm{Si}+{ }^{206} \mathrm{Hg}$ & 91.029 & $>25.5$ & 26.140 & 26.48 \\
\hline${ }^{241} \mathrm{Am} \rightarrow{ }^{34} \mathrm{Si}+{ }^{207} \mathrm{Bi}$ & 93.926 & $>24.4$ & 25.778 & 26.25 \\
\hline
\end{tabular}

play a key role for the preformation mechanism. The introduction of the proximity forces lower the barrier of cluster emission much more than that for $\alpha$ decay since the asymmetry is weaker. The law according to which the preformation factors follow a simple dependence on 
TABLE III: Predicted half-lives of cluster decay from medium mass nuclei.

\begin{tabular}{|c|c|c|}
\hline$\overline{\text { Decay }}$ & $\overline{Q(\mathrm{MeV})}$ & $\log _{10} T(\mathrm{~s})$ \\
\hline${ }^{114} \mathrm{Ba} \rightarrow{ }^{12} \mathrm{C}+{ }^{102} \mathrm{Sn}$ & 19.05 & 11.12 \\
\hline${ }^{114} \mathrm{Ba} \rightarrow{ }^{16} \mathrm{O}+{ }^{98} \mathrm{Cd}$ & 26.50 & 15.38 \\
\hline${ }^{115} \mathrm{Ba} \rightarrow{ }^{12} \mathrm{C}+{ }^{103} \mathrm{Sn}$ & 18.24 & 13.55 \\
\hline${ }^{115} \mathrm{Ba} \rightarrow{ }^{16} \mathrm{O}+{ }^{99} \mathrm{Cd}$ & 25.88 & 17.30 \\
\hline${ }^{116} \mathrm{Ba} \rightarrow{ }^{12} \mathrm{C}+{ }^{104} \mathrm{Sn}$ & 17.22 & 15.50 \\
\hline${ }^{116} \mathrm{Ba} \rightarrow{ }^{16} \mathrm{O}+{ }^{100} \mathrm{Cd}$ & 24.72 & 18.88 \\
\hline${ }^{117} \mathrm{Ba} \rightarrow{ }^{12} \mathrm{C}+{ }^{105} \mathrm{Sn}$ & 16.27 & 18.70 \\
\hline${ }^{117} \mathrm{Ba} \rightarrow{ }^{16} \mathrm{O}+{ }^{101} \mathrm{Cd}$ & 23.54 & 22.30 \\
\hline${ }^{118} \mathrm{Ba} \rightarrow{ }^{12} \mathrm{C}+{ }^{106} \mathrm{Sn}$ & 15.43 & 20.85 \\
\hline${ }^{118} \mathrm{Ba} \rightarrow{ }^{16} \mathrm{O}+{ }^{102} \mathrm{Cd}$ & 22.12 & 25.30 \\
\hline${ }^{119} \mathrm{Ba} \rightarrow{ }^{12} \mathrm{C}+{ }^{107} \mathrm{Sn}$ & 14.34 & 25.12 \\
\hline${ }^{119} \mathrm{Ce} \rightarrow{ }^{16} \mathrm{O}+{ }^{103} \mathrm{Sn}$ & 27.69 & 16.17 \\
\hline${ }^{120} \mathrm{Ce} \rightarrow{ }^{16} \mathrm{O}+{ }^{104} \mathrm{Sn}$ & 26.58 & 17.75 \\
\hline${ }^{121} \mathrm{La} \rightarrow{ }^{12} \mathrm{C}+{ }^{109} \mathrm{Sb}$ & 13.86 & 28.45 \\
\hline${ }^{121} \mathrm{Ce} \rightarrow{ }^{16} \mathrm{O}+{ }^{105} \mathrm{Sn}$ & 25.49 & 20.48 \\
\hline${ }^{122} \mathrm{Ce} \rightarrow{ }^{16} \mathrm{O}+{ }^{106} \mathrm{Sn}$ & 24.43 & 22.29 \\
\hline${ }^{124} \mathrm{Ce} \rightarrow{ }^{16} \mathrm{O}+{ }^{108} \mathrm{Sn}$ & 22.02 & 28.63 \\
\hline${ }^{125} \mathrm{Pr} \rightarrow{ }^{16} \mathrm{O}+{ }^{109} \mathrm{Sb}$ & 23.09 & 27.98 \\
\hline
\end{tabular}

the mass of the clusters was confirmed. Predictions have been made for some possible decays from medium mass nuclei.
This work was supported by the Natural Science Foundation of China (Grants 10775061, 10505016, 10575119 and 10805016), by the Fundamental Research Fund for Physics and Mathematics of Lanzhou University (LZULL200805), the CAS Knowledge Innovation Project No. KJCX-SYW-N02, and the Major State Basic Research Developing Program of China (2007CB815004).
[1] A. Sandulescu, D. N. Poenaru, and W. Greiner, Sov. J. Part. Nucl. 11, 528 (1980).

[2] H. J. Rose, and G. A. Jones, Nature (London) 307, 245 (1984).

[3] S. Kumar, M. Balasubramaniam, R. K. Gupta, G. Münzenberg, and W. Scheid, J. Phys. G: Nucl. Part. Phys 29, 625 (2003).

[4] R. K. Gupta, S. Dhaulta, R. Kumar, M. Balasubramaniam, G. Münzenberg, W. Scheid, Phys. Rev. C 68, 034321 (2003).

[5] S. Kumar, R. Rani and R. Kumar, J. Phys. G: Nucl. Part. Phys 36, 015110 (2009).

[6] M. Balasubramaniam, and R. K. Gupta, Phys. Rev. C 60 , 064316(1999).

[7] D. N. Poenaru, M. Ivascu, A. Sandulescu and W. Greiner, J. Phys. G: Nucl. Part. Phys. 10, L183 (1984).

[8] W. Greiner, M. Ivascu, D. N. Poenaru and A. Sandulescu, Z. Phys. A 320, 347 (1985).

[9] B. Buck and A. C. Merchant, J. Phys. G: Nucl. Part. Phys. 15, 615 (1989).

[10] G. Royer, R. K. Gupta, V. Y. Denisov, Nucl. Phys. A 632, 275 (1998).

[11] G. Royer, R. Moustabchir, Nucl. Phys. A 683, 182 (2001).

[12] K. P. Santhosh, R. K. Biju and A. Joseph, J. Phys. G: Nucl. Part. Phys 35, 085102 (2008).

[13] K. P. Santhosh, R. K. Biju, J. Phys. G: Nucl. Part. Phys
36, 015107 (2009).

[14] J. M. Dong, H. F. Zhang, J. Q. Li and W. Scheid, Eur. Phys. J. A 41, 197 (2009).

[15] F. R. Xu, J. C. Pei, Phys. Lett. B 642, 322 (2006).

[16] G. Audi, A. H. Wapstra and C. Thibault, Nucl. Phys. A 729, 337 (2003).

[17] H. F. Zhang and G. Royer, Phys. Rev. C 77, 054318 (2008).

[18] Madhubrata Bhattacharya and G. Gangopadhyay, Phys. Rev. C 77, 027603 (2008).

[19] K. Varga, R. G. Lovas, and R. J. Liotta, Phys. Rev. Lett. 69, 37 (1992).

[20] P. Mohr, Phys. Rev. C 61, 045802 (2000).

[21] R. Blendowske and H. Walliser, Phys. Rev. Lett. 61, 1930 (1988).

[22] D. N. Poenaru, Y. Nagame, R. A. Gherghescu, and W. Greiner, Phys. Rev. C 65, 054308 (2002).

[23] D. N. Poenaru, W. Greiner, R. A. Gherghescu, Phys. Rev. C 47, 2030 (1993); D. N. Poenaru, W. Greiner, E. Hourani, Phys. Rev. C 51, 594 (1995).

[24] S. Kumar, D. Bir, R. K. Gupta, Phys. Rev. C 51, 1762 (1995).

[25] G. Shanmugam, G. M. CarmelVigilaBai, B. Kamalaharan, Phys. Rev. C 51, 2616 (1995).

[26] A. Guglielmetti et al., Phys. Rev. C 56, R2912 (1997). 